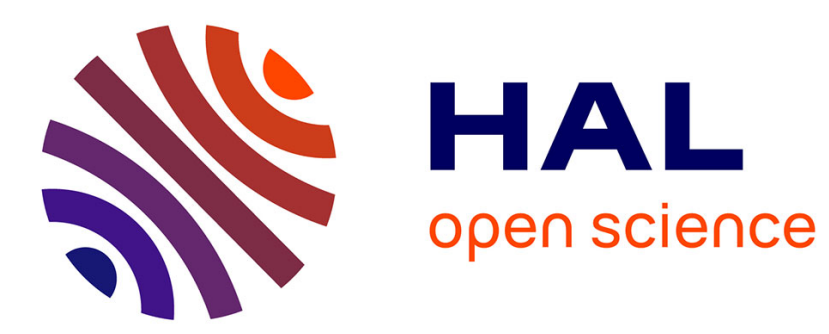

\title{
Study of different strategies for splitting variables in multidisciplinary topology optimization
}

Rajae Aboulaich, Abderrahmane Habbal, Noureddine Moussaid

\section{To cite this version:}

Rajae Aboulaich, Abderrahmane Habbal, Noureddine Moussaid. Study of different strategies for splitting variables in multidisciplinary topology optimization. 2011. hal-00648701

HAL Id: hal-00648701

https://hal.inria.fr/hal-00648701

Preprint submitted on 6 Dec 2011

HAL is a multi-disciplinary open access archive for the deposit and dissemination of scientific research documents, whether they are published or not. The documents may come from teaching and research institutions in France or abroad, or from public or private research centers.
L'archive ouverte pluridisciplinaire HAL, est destinée au dépôt et à la diffusion de documents scientifiques de niveau recherche, publiés ou non, émanant des établissements d'enseignement et de recherche français ou étrangers, des laboratoires publics ou privés. 


\title{
Study of different strategies for splitting variables in multidisciplinary topology optimization
}

\section{R. Aboulaich ${ }^{a}{ }^{1}$, A. Habbal ${ }^{b}$ and N. Moussaid ${ }^{a, c}$}

${ }^{a}$ LERMA, Ecole Mohammadia d'Ingénieurs, Avenue Ibn Sina B.P 765, Agdal, Rabat.

${ }^{b}$ LJAD, Université de Nice Sophia-Antipolis, Valrose, 06108 Nice Cedex 2, France. ${ }^{c}$ Université Cadi Ayyad, École Supérieure de Technologie d'Essaouira, Maroc.

\begin{abstract}
This paper is devoted to the minimization of the thickness of an elastic structure under competitive loadings. We propose to determine an equilibrium thickness using game theory. We consider two loads exercised separately on two parts of the plate and we aim to optimize both compliances so we deal with a multiloading optimization problem. Firstly, the design variable is taken to be the thickness of the plate. In a second step, we assume that the thickness depends on two independent functions, that we consider as strategies. The multidisciplinary optimization problem is solved as a non-cooperative game and we determine a Nash equilibrium. Finally, some numerical simulations are presented and discuted.
\end{abstract}

keywords: Multidisciplinary topology optimization, Variable thickness, Game theory, Nash Equilibrium, finite element method, FreeFem++.

AMS Subject Classification: 35R30, 91A10.

\section{Introduction}

Structural optimization has important applications in large fields of applied sciences and engineering, and has taken more attention from many researchers and engineers in the last years. Related computational optimization methods have been received considerable attention in the recent decades. By introducing the techniques of topology optimization to the design of continuum structures (Bendsøe, et al. (1988)), these methods have been applied with success to a variety of types

\footnotetext{
${ }^{1}$ Corresponding author. E-mail: aboulaich@emi.ac.ma
} 
of structural design problems. In general, structural optimization problems are of three classes, including size, shape and topology optimization, for thorough details see for instance (Allaire, 2007; Allaire, 2005). In this work, we consider the optimal design of a plate of variable thickness $h$. The theme of this work stems from (Allaire 2005), where the optimal design of the plate is considered. The aim of this paper is to use a game theory approach to determine the optimal thickness of a plate subjected to two loads exercised separately, on two parts of the plate. Habbal, (2005) solved a multidisciplinary optimization problem using a non-cooperative game (Nash Game) where the strategy of the players is naturally defined.

In this paper, we firstly consider the state variable to be the plate's thickness. Secondly, we assume that the thickness depends on design strategies of the material $s$ and $t$. Hence, we obtain a multidisciplinary optimization problem. To determine the optimal thickness as Nash equilibrium, we will use in both cases two criteria $j_{s}$ and $j_{t}$ associated with the two players respectively. We use, in this case, a concurrent optimization realized by an algorithm which solves the Nash game between the two players. The two players act following different objectives; in particular, player 1 has to choose his strategies in order to minimize his function $j_{s}$, while player 2 has to minimize the function $j_{t}$. To use this optimization technique, we study different strategies for splitting variables in topology optimization (see section 3).

\section{Setting of the problem}

Within the framework of linear elasticity, under the plane strain assumptions, we consider a flat two-dimensional plate section $\Omega$ of variable thickness $h(x)$ (see figure 1).

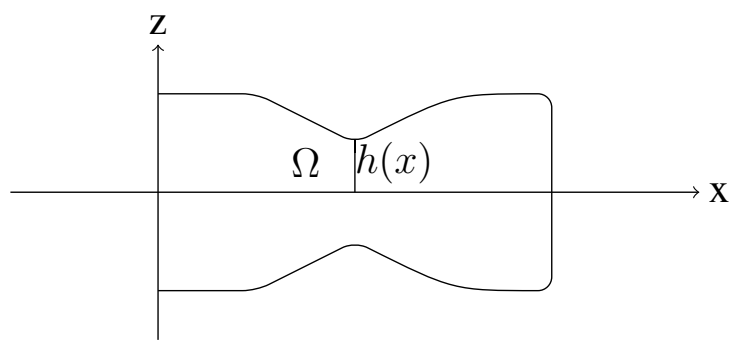

Figure 1: Flat plate of variable thickness in the $x$ direction only

The boundary of $\Omega$ is made of three disjoint parts $\partial \Omega=\Gamma_{D} \cup \Gamma_{N} \cup \Gamma$, with Dirichlet boundary conditions on $\Gamma_{D}$, and Neumann boundary conditions on $\Gamma_{N} \cup \Gamma$. The boundary part $\Gamma_{D}$ is supposed to be fixed, while $\Gamma_{N}$ is submitted to a $g$ surface load and $\Gamma$ is free of any load(see figure 2). 
Figure 2: Boundary conditions for an elastic plate: single load case

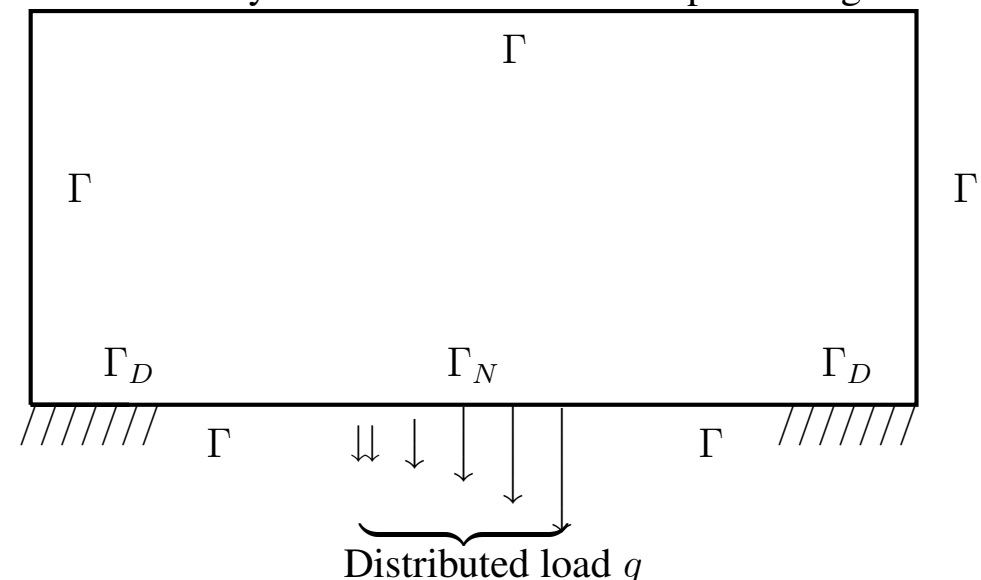

The displacement $u \in H^{1}(\Omega)$ is the solution of the linear elasticity system

$$
\left\{\begin{aligned}
-\operatorname{div} \sigma & =0 \quad \text { in } \Omega \\
\sigma & =2 \mu h e(u)+\lambda h t r(e(u)) I, \quad I=\text { Identity Matrix } \\
u & =0 \quad \text { on } \Gamma_{D} \\
\sigma . n & =g \quad \text { on } \Gamma_{N} \\
\sigma . n & =0 \quad \text { on } \Gamma
\end{aligned}\right.
$$

where $g \in\left(H^{-1 / 2}\left(\Gamma_{N}\right)\right)^{2}$ is a given surface load, $n$ is the outward normal to the boundary, $\sigma$ is the associated stress tensor, which is related via Hooke's law to the linearized strain tensor $e(u)$ via

$$
\sigma=2 \mu h e(u)+\lambda h \operatorname{tr}(e(u)) I
$$

The linearized strain tensor $e(u)$ is given by

$$
e(u)=\frac{1}{2}\left(\nabla u+(\nabla u)^{T}\right)=\frac{1}{2}\left(\frac{\partial u_{i}}{\partial x_{j}}+\frac{\partial u_{j}}{\partial x_{i}}\right)_{1 \leq i, j \leq 2}
$$

$t r$ denotes the trace of a matrix, and $\lambda, \mu$ are Lame coefficients related to Young's modulus $E$ and to the Poisson ratio $\nu$ by:

$$
\mu=\frac{E}{2(1+\nu)} \quad \lambda=\frac{E \nu}{(1-2 \nu)(1+\nu)}
$$

We aim to optimize the plate by varying its thickness $h$ which is limited by minimal values $h_{\min }$ and maximal ones $h_{\max }$, in the admissible set defined by

$$
\mathcal{U}_{h}=\left\{h \in L^{\infty}(\Omega), \quad h_{\min } \leq h \leq h_{\max } \text { a.e in } \Omega \quad \int_{\Omega} h(x) d x=h_{0}|\Omega|\right\}
$$

where, $h_{0}$ is an imposed mean thickness $h_{\min } \leq h_{0} \leq h_{\max }$. It is easy to construct a projection operator for each of these constraints taken separately, so the projection operator is:

$$
(P(h))=\max \left(h_{\min }, \min \left(h_{\max }, h\right)\right) .
$$




\section{Thickness optimization of an elastic plate}

We recall classical results from the literature. Let us consider the compliance minimization problem :

$$
\min _{h} j(h)
$$

where

$$
j(h)=\int_{\Gamma_{N}} g u d s, \text { where } u \text { solves: }(2.1)
$$

For a given $h>0$ there exists a unique solution $u$ of (2.1) in the space $V$, where

$$
V=\left\{v \in H^{1}(\Omega)^{2} \text { such that } v=0 \text { on } \Gamma_{D}\right\}
$$

Théorème 1. The problem (3.1) admits at least one optimal solution.

Proof. As $u$ is a solution of problem (2.1), then $u$ is the unique solution of the following minimization problem:

$$
J(v)=\left\{\frac{1}{2} \int_{\Omega}\left(2 \mu h|e(v)|^{2}+\lambda h|d i v v|^{2}\right) d x-\int_{\Gamma_{N}} g v d s\right\}
$$

i.e.,

$$
\begin{aligned}
\min _{v} J(v) & =\frac{1}{2} \int_{\Omega}\left(2 \mu h|e(u)|^{2}+\lambda h|d i v u|^{2}\right) d x-\int_{\Gamma_{N}} g u d s \\
& =-\frac{1}{2}\left(\int_{\Gamma_{N}} g u d s\right) \\
& =-\frac{1}{2} j(h)
\end{aligned}
$$

then,

$$
j(h)=2 \max _{v}\left\{\int_{\Gamma_{N}} g v d s-\frac{1}{2} \int_{\Omega}\left(2 \mu h|e(v)|^{2}+\lambda h|d i v v|^{2}\right) d x\right\}
$$

It is then a supremum envelop of continuous affine with respect to the variable $h$, so it is convex and lower semicontinuous.

As a convex function over $\mathcal{U}_{h}$, it is also weak-* lower semicontinuous. Since the set $\mathcal{U}_{h}$ is weak-* compact, there exists a minimum of $j(h)$ over $h$.

We use the Lagrange multiplier method to derive an optimality system of equations from which solutions of the optimization problem (3.1) may be determined.

Let $u, v \in V$, we define the Lagrangian

$$
\mathcal{L}(u, v, h)=\int_{\Gamma_{N}} \text { g.uds }-\int_{\Omega}(2 h \mu e(u) \cdot e(v)+h \lambda d i v u d i v v) d x+\int_{\Gamma_{N}} \text { g.vds } .
$$

Setting to zero the first variations with respect to the multiplier $v$ yield the constraints

$$
<\frac{\partial \mathcal{L}}{\partial v}(u, v, h), \Phi>=-\int_{\Omega}(2 h \mu e(u) . e(\Phi)+h \lambda d i v u d i v \Phi) d x+\int_{\Gamma_{N}} g . \Phi d s .
$$


Setting to zero the first variations with respect to $u$ yield the adjoint equations

$$
<\frac{\partial \mathcal{L}}{\partial u}(u, v, h), \Phi>=-\int_{\Omega}(2 h \mu e(\Phi) . e(v)+h \lambda \operatorname{divvdiv} \Phi) d x+\int_{\Gamma_{N}} g . \Phi d s
$$

By combining the results we get

$$
<\frac{\partial \mathcal{L}}{\partial h}(u, v, h), w>=-\int_{\Omega}(2 \mu e(u) \cdot e(v)+\lambda \text { divvdivu }) \cdot w d x
$$

then

$$
\nabla j(h)=-(2 \mu e(u) \cdot e(v)+\lambda d i v v d i v u)
$$

The topology design of a plate is investigated, a surface load is applied to the plate $g=$ $(0,-100)$. The domain $\Omega$ is $]-1,1[\times] 0,1$. The material response is given by equation (2.2) with a Young's modulus $E=100$ and Poisson's ratio $\nu=0.3$. The upper thickness is $h_{\max }=1$ and the lower thickness is $h_{\max }=0.001$.

The procedure described above does not require any great programming efforts in order to solve the compliance topology design problem. In the case of compliance optimization, the state or displacements $u$ is the solution of the linear elasticity equation (2.1). We use $P 2 \times P 2$ Lagrange finite elements to compute $u$, using FreeFem++, the thickness $h$ is approximated by means of piecewise-constant interpolation. The results are depicted on Figures 3 and 4.

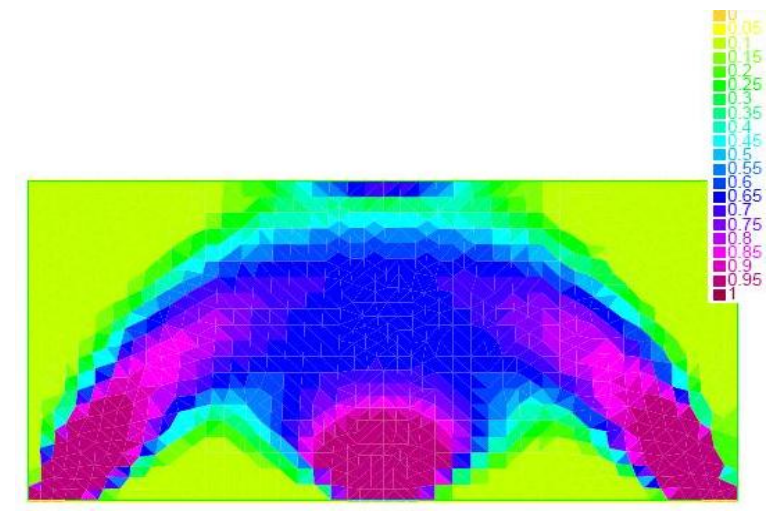

Figure 3: Optimal plate

We remark that we have obtained a composite structure, i.e. the thickness values vary in the interval $\left[h_{\min }, h_{\max }\right]$, to find feasible ones we use a penalty technique to force the thickness to take only value 0 or 1 . For that, we redo some iterations of minimization using the thickness penalized.

$$
h_{p e n}=\frac{1-\cos \left(\pi h_{\text {opt }}\right)}{2}
$$

where $h_{\text {opt }}$ is the thickness values obtained after convergence and $h_{p e n}$ is the thickness value penalized. 
We present, in figure 4, the optimal plate obtained with a penalization techniques. Figure 5 present the evolution of the cost function.

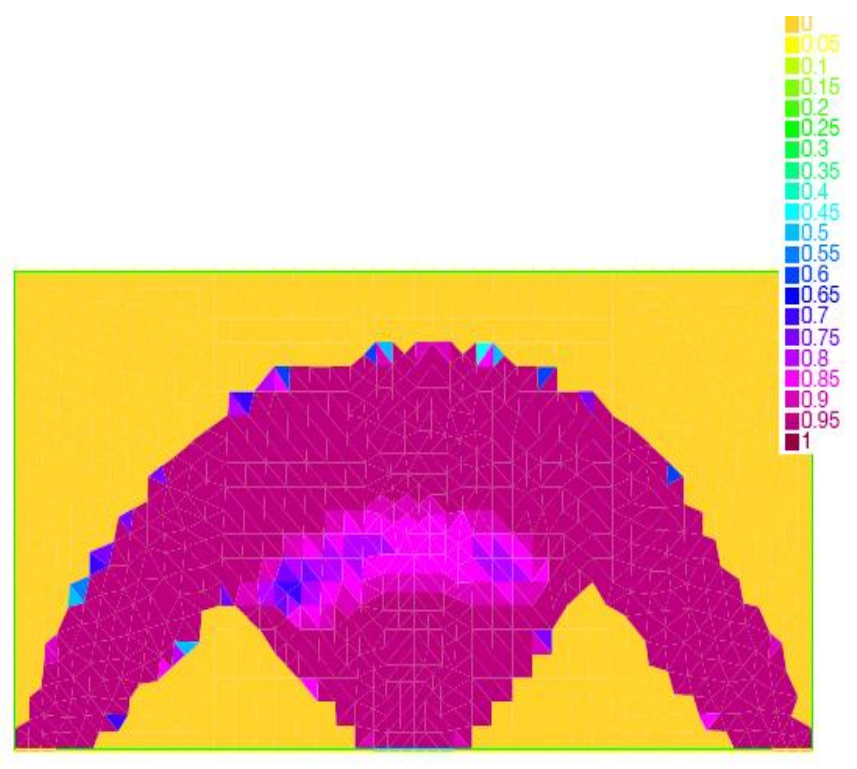

Figure 4: Optimal plate obtained using the penalization technique

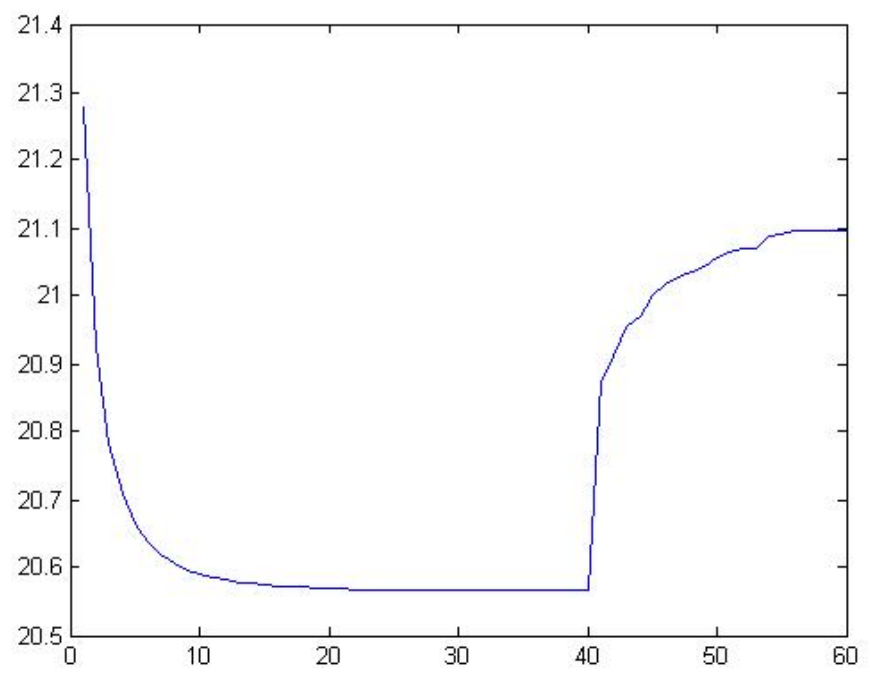

Figure 5: Convergence history of the compliance

The convergence is obtained in less than 40 iterations (see figure 5) for computing $h_{\text {opt }}$ and 
from 60th iteration for $h_{\text {pen }}$. The penalization phase took 20 more iterations.

The framework described for minimum compliance for a single load case generalizes easily to the situation where design for multiple load conditions is formulated as a minimization of a weighted average of the compliances for each of the load cases as a Nash game.

\section{Split of thickness in concurrent optimization}

In the following section, we suppose that the plate is subject to two boundary conditions (multiple loads) $g_{s} \in\left(H^{-1 / 2}\left(\Gamma_{N s}\right)\right)^{2}$ and $g_{t} \in\left(H^{-1 / 2}\left(\Gamma_{N t}\right)\right)^{2}$ at the part $\Gamma_{N s}$ and $\Gamma_{N t}$, such that $\Gamma_{N s} \cup \Gamma_{N t}=$ $\Gamma_{N}$ (see figure 6).

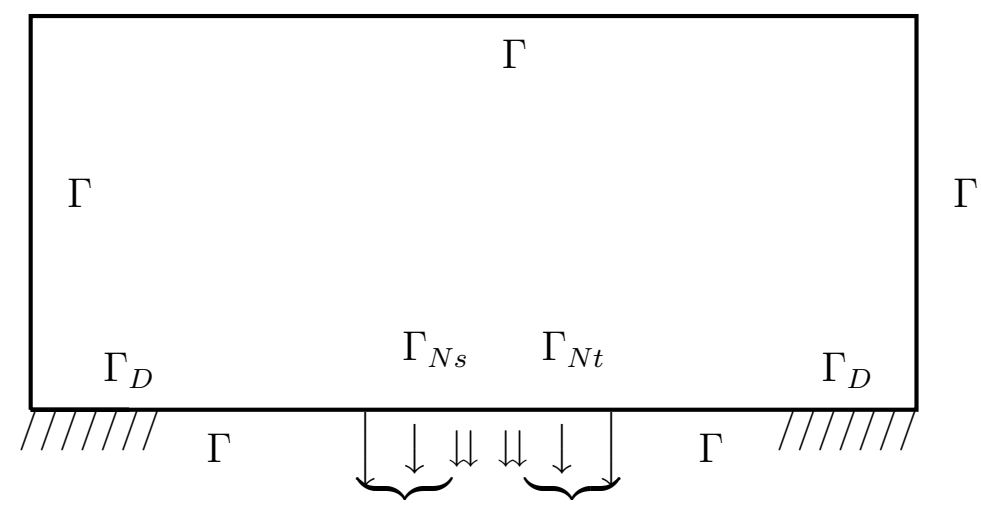

Figure 6: Concurrent load case

\subsection{Variable thickness}

We split the original design variable (thickness $h$ ) into two strategies (Aboulaich et al. (2010a); Aboulaich et al. (2010b)), formally we denote $h=(X, Y)$ such that $X, Y \in \mathcal{U}_{h}$. The split of the variable $h$ is to construct two allocation tables $\mathrm{P}$ and $\mathrm{Q}$ in $\{0,1\}^{n}$, where $P_{i}+Q_{i}=1,1 \leq i \leq n$ and $n$ is the size of $h$. Let $I_{12}=\{1, \ldots, n\}$ be a set of indices of cardinality $n, I_{1}$ a subset of $I_{12}$ of cardinality $n_{1}$, and $I_{2}$ its complement of cardinality $n_{2}$, that is to say $I_{12}=I_{1} \cup I_{2}$.

Suppose that:

$$
\left\{\begin{array}{l}
X=\left(h_{i}\right), \text { for } i \in I_{1}, \\
Y=\left(h_{i}\right), \text { for } i \in I_{2} .
\end{array}\right.
$$

We define in this case the integer allocation table $P$ of size $n$ :

$$
P_{i}=1, \forall i \in I_{1}, P_{i}=0, \forall i \in I_{2}
$$

so that

$$
h=P . h+(\mathcal{I}-P) . h=(X, Y) \quad \text { where } \mathcal{I}=(1, \ldots, 1) .
$$


Where "." denote the Hadamard product (i.e. $\left.(P . h)_{i}=P_{i} h_{i}, P . h \in \mathbb{R}^{n}\right)$, and $(X, Y)$ is defined in equation (4.1).

We consider the functionals $j_{1}(X, Y)$ and $j_{2}(X, Y)$ defined by:

$$
j_{1}(X, Y)=\int_{\Gamma_{N s}} g_{s} u_{1} d s
$$

where $u_{1}$ is the solution of the problem $\left(P_{1}\right)$ :

$$
\left(P_{1}\right)\left\{\begin{aligned}
-\operatorname{div} \sigma_{1} & =0 \quad \text { in } \Omega \\
\sigma_{1} & =2 \mu X e\left(u_{1}\right)+\lambda X \operatorname{tr}\left(e\left(u_{1}\right)\right) I \\
u_{1} & =0 \quad \text { on } \Gamma_{D} \\
\sigma_{1} \cdot n & =g_{s} \quad \text { on } \Gamma_{N s} \\
\sigma_{1} \cdot n & =0 \quad \text { on } \Gamma_{N t} \cup \Gamma
\end{aligned}\right.
$$

and

$$
j_{2}(X, Y)=\int_{\Gamma_{N t}} g_{t} u_{2} d s
$$

where $u_{2}$ is the solution of the problem $\left(P_{2}\right)$ :

$$
\left(P_{2}\right)\left\{\begin{aligned}
-d i v \sigma_{2} & =0 \quad \text { in } \Omega \\
\sigma_{2} & =2 \mu Y e\left(u_{2}\right)+\lambda Y \operatorname{tr}\left(e\left(u_{2}\right)\right) I \\
u_{2} & =0 \quad \text { on } \Gamma_{D} \\
\sigma_{2} \cdot n & =g_{t} \quad \text { on } \Gamma_{N t} \\
\sigma_{2} \cdot n & =0 \quad \text { on } \Gamma_{N s} \cup \Gamma
\end{aligned}\right.
$$

We minimize $j_{1}$ and $j_{2}$ by acting on $\mathcal{U}_{h}$, until the convergence to a Nash equilibrium $h_{N E}$. In a competitive game, the two players act following different objectives; in particular, player 1 have to choose his strategies in order to minimize his function $j_{1}$, while player 2 has to minimize the function $j_{2}$.

We say that the couple $\left(X^{\star}, Y^{\star}\right)$ is a point of Nash equilibrium, if and only if

$$
(P)\left\{\begin{array}{l}
j_{1}\left(X^{\star}, Y^{\star}\right)=\min _{X} j_{1}\left(X, Y^{\star}\right), \\
j_{2}\left(X^{\star}, Y^{\star}\right)=\min _{Y} j_{2}\left(X^{\star}, Y\right),
\end{array}\right.
$$

i.e., when a player can not improve its criteria over the other, it means that the system reaches a state equilibrium called Nash equilibrium.

Solving the Nash equilibrium requires solving the following two problems, namely

$$
\min _{X} j_{1}(X, Y) \text { and } \min _{Y} j_{2}(X, Y)
$$

The Nash equilibrium is computed by the following decomposition algorithm.

Set $n=0$. Starting from an initial design pair $h^{(0)}=\left(X^{(0)}, Y^{(0)}\right)$. 
Step 1:

Phase 1: solve the problem

$$
\min _{X} j_{1}\left(X, Y^{(n)}\right) \rightarrow X^{(n+1)}
$$

Phase 2: solve the problem

$$
\min _{Y} j_{2}\left(X^{(n)}, Y\right) \rightarrow Y^{(n+1)}
$$

Step 2: set $h^{(n+1)}=\left(X^{(n+1)}, Y^{(n+1)}\right)$ until convergence, redo the parallel phases 1 and 2 .

The phases 1 and 2 are solved by the finite element method software; FreeFem++.

In the case of compliance optimization, the state or displacement $u_{1}$ and $u_{2}$ are the solution of the linear elasticity problems $\left(P_{1}\right)$ and $\left(P_{2}\right)$. We use the package of $\mathrm{G}$. Allaire written in Freefem++, for more details see (Allaire, 2005). We propose the Lagrange $P 2 \times P 2$ Finite element method to numerically solve the problem $\left(P_{1}\right)$ and $\left(P_{2}\right)$, thickness $h_{1}$ and $h_{2}$ are approximated by means of piecewise-constant interpolation. We will take in this simulation the indices in $I_{1}$ are odd while the indices in $I_{2}$ are even. The figure 7 presents the obtained optimal shape by using Nash games between the variables of the odd indices and the ones of the even indices. For the numerical simulation we use the following parameters

$$
g_{s}=g_{t}=(0,-100)
$$

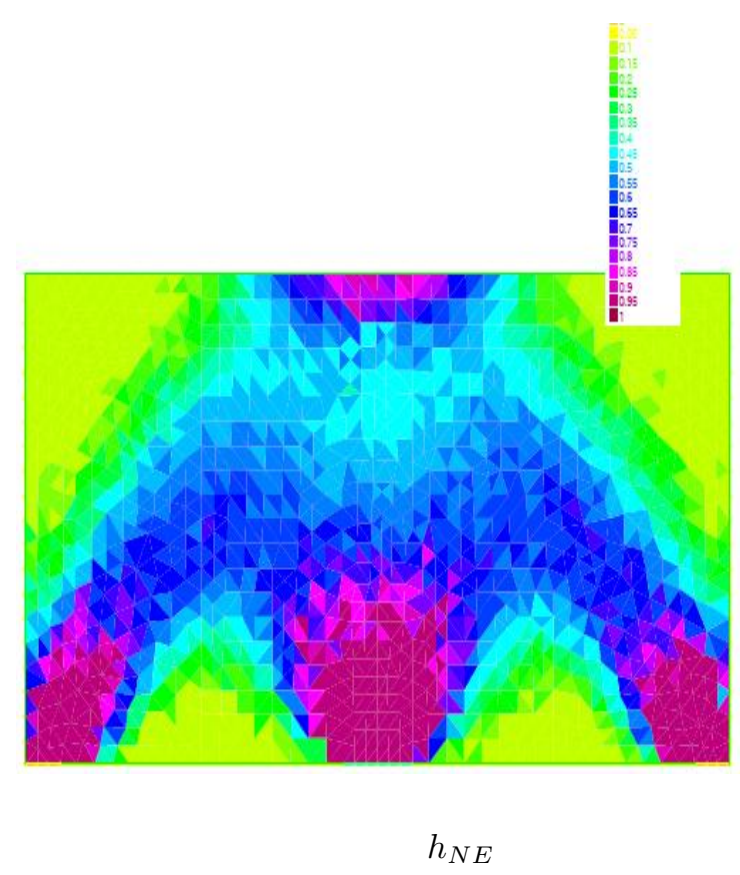

Figure 7: Optimal shape of the plate 
The resulting optimal design being composite, it is then "projected" on the set of classical shapes by applying again the previous scheme with the following slight modification: the thickness is updated setting $h^{(n+1)}=h_{p e n}$ (see Allaire, 2007), where

$$
h_{\text {pen }}=\frac{1-\cos \left(\pi h^{(n+1)}\right)}{2}
$$

instead of $h^{(n+1)}=h_{N E}$.

Figure 8 depicts the optimal plate obtained after penalization with Nash equilibrium approach. The Nash overall scheme converged after 3 iterations.

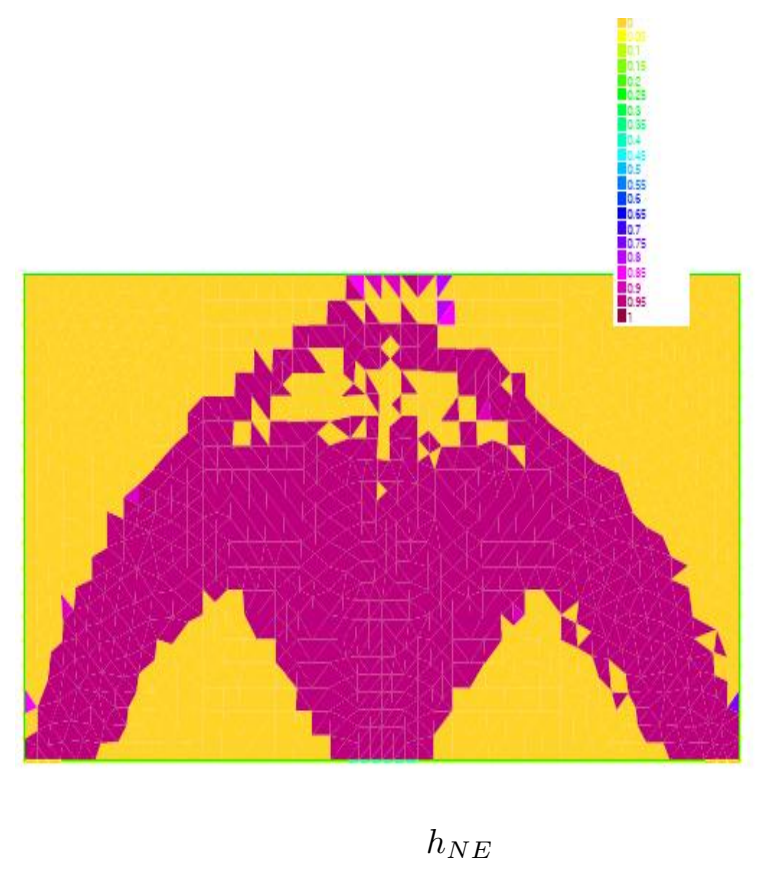

Figure 8: Optimal shape of the plate obtained after penalization

We remark that the obtained optimal forme is not quite similar to that obtained by minimizing the function $j$, hence our splitting is ill-chosen. To avoid using this splitting, we propose to use another technique of thickness splitting. We assume that the thickness depends on two functions $s$ and $t$. We determine the Nash equilibrium between the two strategies. 


\subsection{Variables strategies of the materials}

We are interested in this section in the case where we have two objective functions. We suppose that the plate thickness depends on strategies $s(x)$ and $t(x)$. This problem will be treated as a concurrent optimization problem, by Nash games between two players using two strategies. The first player minimizes his objective function using the first strategy $s(x)$, the second player, using the second one $t(x)$. We shall study the following forms :

$$
\left\{\begin{array}{l}
h(s, t)=s t \\
h(s, t)=\frac{1}{2} s+\frac{1}{2} t \\
h(s, t)=s(1-t)+t(1-s)
\end{array}\right.
$$

These expressions many be summarized as follows : $h(s, t)=a s t+b s+c t$ (respectively $a=$ $1, b=0, c=0 ; a=0, b=\frac{1}{2}, c=\frac{1}{2}$ and $\left.a=-2, b=1, c=1\right)$.

For example, the choice of 1 ) is motivated by the requirement that presence of equilibrium material needs the conjunction of presence at optimal material for $j_{1}$ and $j_{2}$.

$A_{i}:$ event $=$ presence of optimal material for $j_{i} ; i=1,2$

$B:$ event $=$ presence of optimal material at equilibrium.

One could interpret $1-3$ in term of probabilities as :

1. $P(B)=P\left(A_{1}\right) P\left(A_{2}\right) \quad\left(B=A_{1} \cap A_{2}\right)$

2. $P(B)=\frac{1}{2} P\left(A_{1}\right)+\frac{1}{2} P\left(A_{2}\right)$

3. $P(B)=P\left(A_{1}\right) P\left(\overline{A_{2}}\right)+P\left(\overline{A_{1}}\right) P\left(A_{2}\right) \quad\left(B=\left(A_{1} \cap \overline{A_{2}}\right) \cup\left(\overline{A_{1}} \cap A_{2}\right)\right)$.

Of course, as generally both the functions depend on the two domains, the strategies of one player influences the choices of the other one: The two players act simultaneously until an equilibrium is found: in that case, each player has minimized his own function with a common pair of strategies. We introduce the following spaces, of admissible solutions of the strategies $s(x)$ and $t(x)$ respectively:

$$
\mathcal{U}_{s}=\left\{s \in L^{\infty}(\Omega), \quad 0 \leq s \leq 1 \text { a.e. in } \Omega\right\}
$$

and

$$
\mathcal{U}_{t}=\left\{t \in L^{\infty}(\Omega), \quad 0 \leq t \leq 1 \text { a.e. in } \Omega\right\}
$$


The optimization problem we want to consider are as follows.

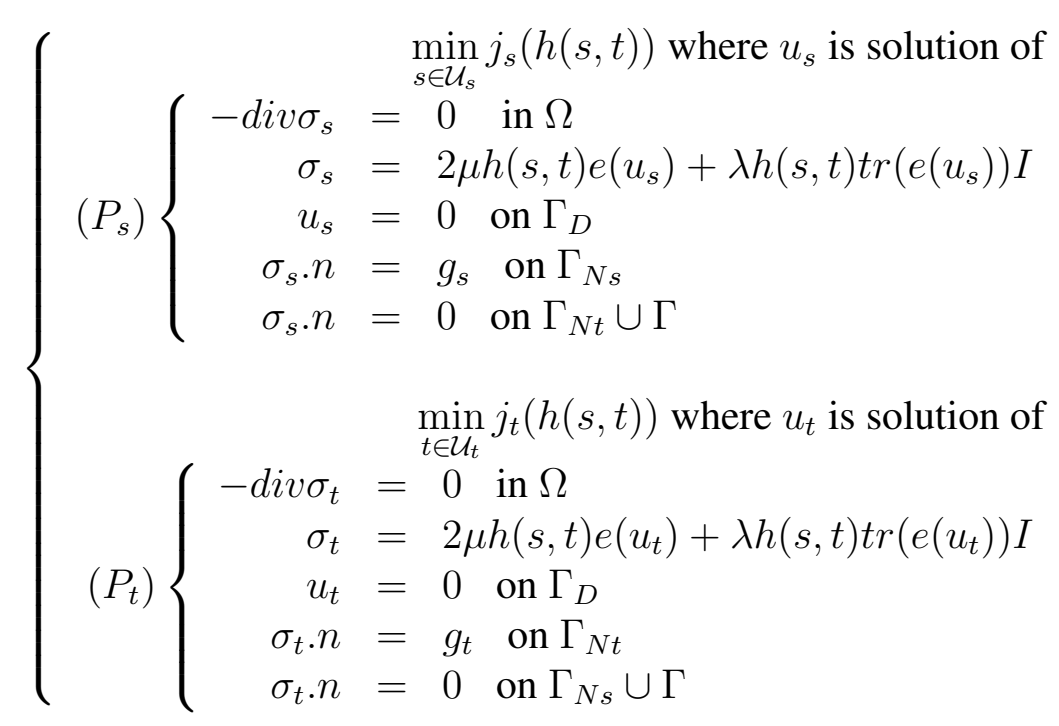

where

$$
j_{s}(s, t)=\int_{\Gamma_{N s}} g_{s} u_{s} d s, \text { and } \Gamma_{N s}=\text { support of } g_{s}
$$

and

$$
j_{t}(s, t)=\int_{\Gamma_{N t}} g_{t} u_{t} d s, \quad \text { and } \quad \Gamma_{N t}=\text { support of } g_{t}
$$

Theorem 1. There exists a Nash equilibrium $\left(s^{\star}, t^{\star}\right) \in \mathcal{U}_{s} \times \mathcal{U}_{t}$ solution of the problem (4.8).

Proof. The sets $\mathcal{U}_{s}$ and $\mathcal{U}_{t}$ are compact convex for the weak topology ${ }^{\star} L^{\infty}$. The functionals $j_{s}$ and $j_{t}$ are convex and lower semicontinuous for the weak topology ${ }^{\star} L^{\infty}$.

In fact, $u_{s}$ is the solution of problem $\left(P_{s}\right)$, so $u_{s}$ is the unique solution of the following minimization problem:

$$
\min _{v}\left\{\frac{1}{2} \int_{\Omega}\left(2 \mu h(s, t)|e(v)|^{2}+\lambda h(s, t)|d i v v|^{2}\right) d x-\int_{\Gamma_{N s}} g_{s} v d s\right\}
$$

whence,

$$
j_{s}(s, t)=2 \max _{v}\left\{\int_{\Gamma_{N s}} g_{s} v d s-\frac{1}{2} \int_{\Omega}\left(2 \mu h(s, t)|e(v)|^{2}+\lambda h(s, t)|d i v v|^{2}\right) d x\right\} .
$$

On the other hand, the function $s \mapsto \int_{\Omega}\left(2 \mu h(s, t)|e(v)|^{2}+\lambda h(s, t)|d i v v|^{2}\right) d x$ is affine with respect to $s$, accordingly, one concludes that it is weak ${ }^{\star}$ lower semicontinuous (see Aubin, 1979), likewise for $j_{t}$. Then we have at least the existence of one Nash equilibrium $\left(s^{\star}, t^{\star}\right)$ (see Aubin, 1979).

For example, a problem considers two objectives to minimize $j_{s}(s, t)$ and $j_{t}(s, t)$ where design variables are $s$ and $t$. the problem will be solved firstly as a Nash games secondly as a Pareto 
optimum. In the Nash games, the player 1 will minimize $j_{s}$ with respect to $s$ where the design variable $t$ is fixed by player 2. Player 2 will only optimize $t$ to minimize $j_{t}$ using design variable $s$ fixed by player 1 .

\section{Algorithm to compute a Nash equilibrium}

Set $n=0$. Starting from an initial design pair $\left(s^{(0)}, t^{(0)}\right)$.

Phase 1: solve the problem

$$
\min _{s} j_{s}\left(s, t^{(n)}\right) \rightarrow s^{(n+1)}
$$

a. Update the local proportion with a step size $\rho_{s}^{(n)}>0$ by

$$
s^{(n+1)}=\min \left(1, \max \left(0, \tilde{s}^{(n+1)}\right)\right) \text { with } \tilde{s}^{(n+1)}=s^{(n)}-\rho_{s}^{(n)} \frac{\partial j_{s}}{\partial s}+\frac{\partial h(s, t)}{\partial s} L_{s}^{(n)},
$$

where $L_{s}^{(n)}$ is the Lagrange multiplier for the volume constraint.

b. Penalization

$$
s^{(n+1)}=\frac{\left(1 .-\cos \left(\pi s^{(n+1)}\right)\right)}{2}
$$

Phase 2: solve the problem

$$
\min _{t} j_{t}\left(s^{(n)}, t\right) \rightarrow t^{(n+1)}
$$

a. Update the local proportion with a step size $\rho_{t}^{(n)}>0$ by

$$
t^{(n+1)}=\min \left(1, \max \left(0, \tilde{t}^{(n+1)}\right)\right) \text { with } \tilde{t}^{(n+1)}=t^{(n)}-\rho_{t}^{(n)} \frac{\partial j_{t}}{\partial t}+\frac{\partial h(s, t)}{\partial t} L_{t}^{(n)},
$$

where $L_{t}^{(n)}$ is the Lagrange multiplier for the volume constraint.

b. Penalization

$$
t^{(n+1)}=\frac{\left(1 .-\cos \left(\pi t^{(n+1)}\right)\right)}{2}
$$

Phase 3:

$$
h(s, t)(x)=a s^{(n+1)} t^{(n+1)}+b s^{(n+1)}+c t^{(n+1)}
$$

$n=n+1$. Go to phase 1 , until convergence.

The volume constraint $\int_{\Omega} h(s, t) d x=h_{0}|\Omega|$ is enforced by adjusting the Lagrange multiplier $L_{s}^{(n)}$ and $L_{t}^{(n)}$ by a simple bisection at each iteration.

We set

$$
j_{w}=w j_{s}(s, t)+(1-w) j_{t}(s, t), w \in[0,1] .
$$

For each $w$ we compute the optima, the set of which forms the Pareto front (at least in the convex case). Our algorithm is an iterative method, structured as follows: 
1. Starting from an initial design pair $\left(s^{(n)}, t^{(n)}\right)$

$$
h^{(n)}=a s^{(n)} t^{(n)}+b s^{(n)}+c t^{(n)}
$$

2. Phase 1: solve the problem

$$
\min _{s} j_{w}\left(s, t^{(n)}\right) \rightarrow s^{(n+1)}
$$

a. Update the local proportion with a step size $\rho_{s}^{(n)}>0$ by

$$
s^{(n+1)}=\min \left(1, \max \left(0, \tilde{s}^{(n+1)}\right)\right) \text { with } \tilde{s}^{(n+1)}=s^{(n)}-\rho_{s}^{(n)} \frac{\partial j_{w}}{\partial s}+\frac{\partial h(s, t)}{\partial s} L_{s}^{(n)}
$$

where $L_{s}^{(n)}$ is the Lagrange multiplier for the volume constraint.

b. Penalization

$$
s^{(n+1)}=\frac{\left(1 .-\cos \left(\pi s^{(n+1)}\right)\right)}{2}
$$

3. Phase 2: solve the problem

$$
\min _{t} j_{w}\left(s^{(n)}, t\right) \rightarrow t^{(n+1)}
$$

a. Update the local proportion with a step size $\rho_{t}^{(n)}>0$ by

$$
t^{(n+1)}=\min \left(1, \max \left(0, \tilde{t}^{(n+1)}\right)\right) \text { with } \tilde{t}^{(n+1)}=t^{(n)}-\rho_{t}^{(n)} \frac{\partial j_{w}}{\partial t}+\frac{\partial h(s, t)}{\partial t} L_{t}^{(n)},
$$

where $L_{t}^{(n)}$ is the Lagrange multiplier for the volume constraint.

b. Penalization

$$
t^{(n+1)}=\frac{\left(1 .-\cos \left(\pi t^{(n+1)}\right)\right)}{2}
$$

4. Phase 3:

$$
h^{(n+1)}=a s^{(n+1)} t^{(n+1)}+b s^{(n+1)}+c t^{(n+1)}
$$

$n=n+1$. Go to phase 1 , until convergence.

The volume constraint $\int_{\Omega} h(s, t) d x=h_{0}|\Omega|$ is enforced by adjusting the Lagrange multiplier $L_{s}^{(n)}$ and $L_{t}^{(n)}$ by a simple bisection at each iteration.

We have tested this algorithm to solve our problem (4.8) where $h$ depend on $s$ and $t$ (4.5) for different values of $a, b$ and $c$, the numerical results give for each case the same optimal shape obtained by the Nash game compared to the weighting objectives method.

In the numerical test,

$$
\Omega=]-1,1[\times] 0,1\left[, g_{s}=g_{t}=(0,-100), \mu=0.3, E=100, \text { volume }=h_{0}|\Omega|=0.5\right. \text {. }
$$




\section{R. Aboulaich et al.}

The results obtained by the different approach (Nash Equilibrium and Pareto optimum) are listed in tables 1,2 and 3.

Figures 10, 13 and 16 present a comparison between the optimal plate obtained with each approach (Nash Equilibrium and Pareto optimum) in line 1, and optimal strategies $s$ and $t$ obtained with Nash equilibrium in line 2.

Figures 11, 14 and 17 presents a comparison between the optimal plate obtained after penalization with each approach (Nash Equilibrium and Pareto optimum) in line 1, and optimal strategies $s$ and $t$ obtained with Nash equilibrium in line 2.

In figure 9 we reproduce the comparison of the Pareto optimum with Nash Equilibrium. We remark that the Nash Equilibrium do not necessarily belong to the set of not-dominated points (Pareto). 


\subsubsection{Case $1: h(s, t)=s t$}

\begin{tabular}{|c|c|c|}
\hline & Nash Equilibrium & Pareto optimum \\
\hline$j_{1}$ & 11.0687 & 10.80287 \\
$j_{2}$ & 11.0699 & 10.91279 \\
\hline
\end{tabular}

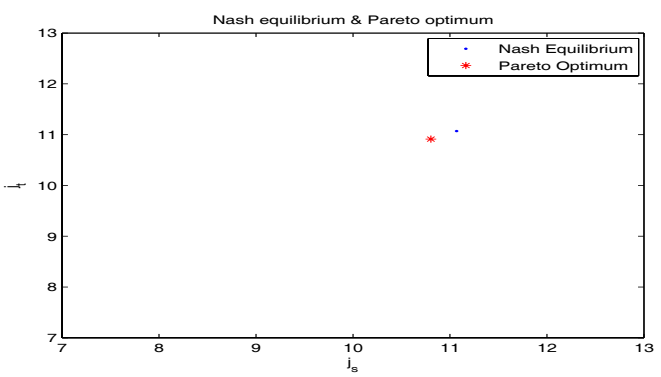

Table 1: Objective function values for Nash equilibrium and Pareto optimum $(\omega=0.5)$.

Figure 9: Pareto optimum and Nash equilibrium

Figure 10: Optimal plate. The Nash overall loop converged in 90 iterations

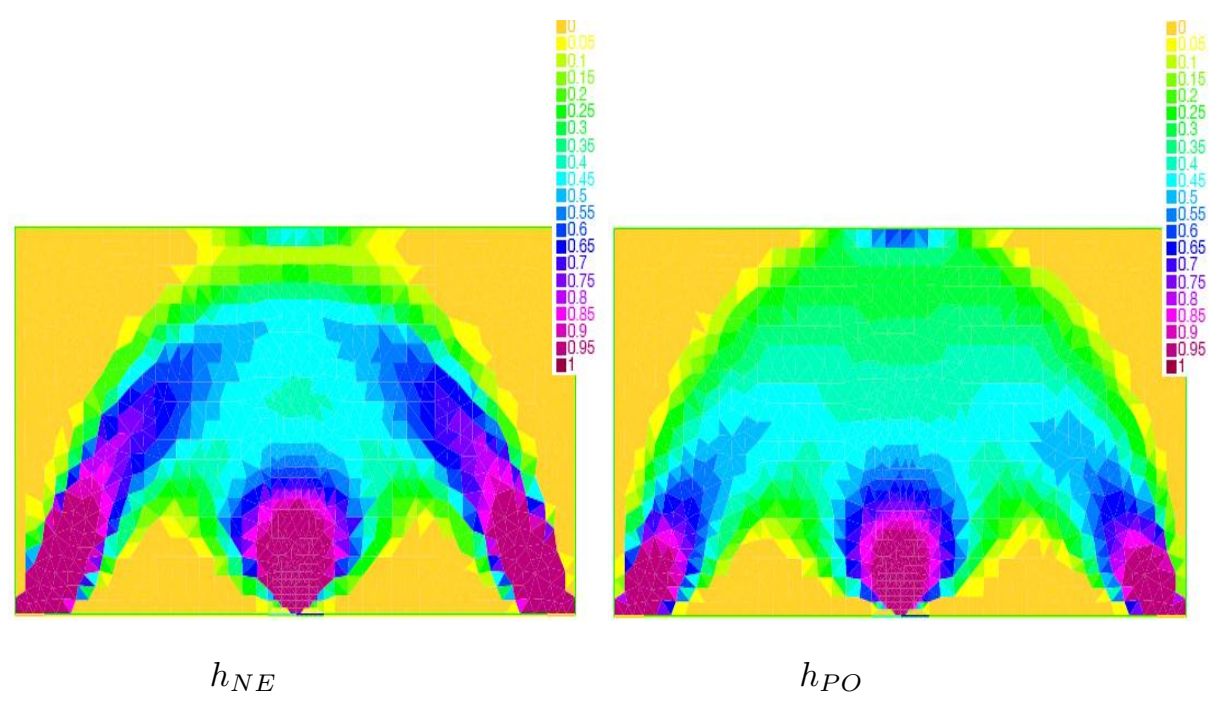

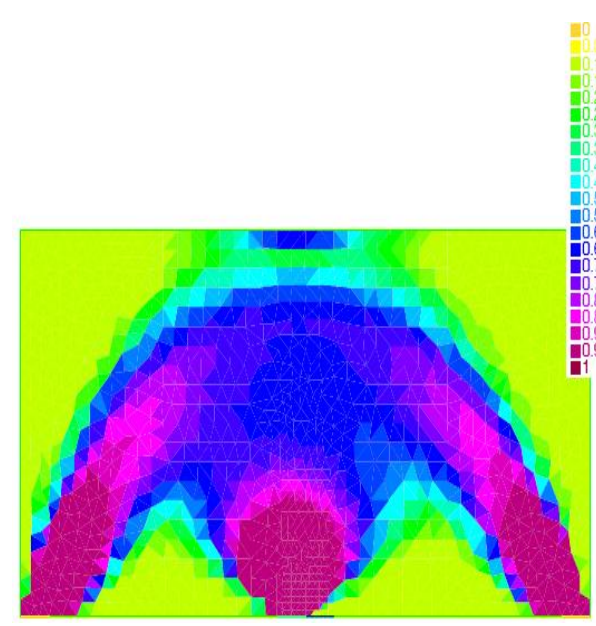

Optimal strategy of $s$

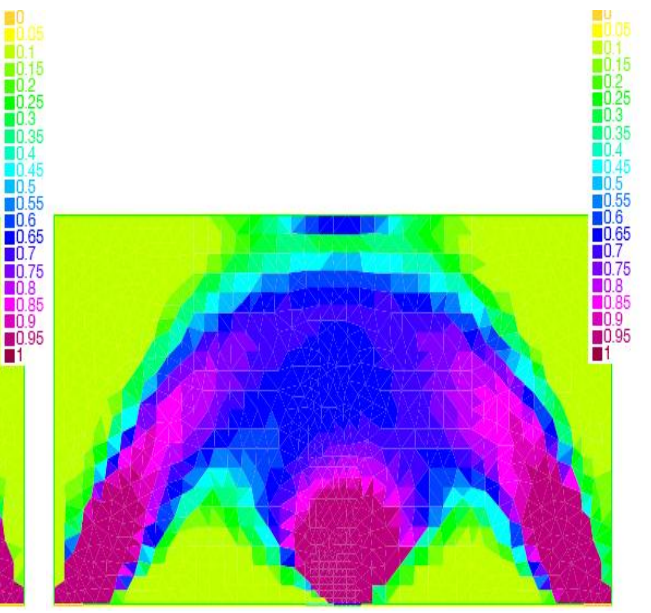

Optimal strategy of $t$ 
Figure 11: Optimal plate. The Nash overall loop converged in 98 iterations
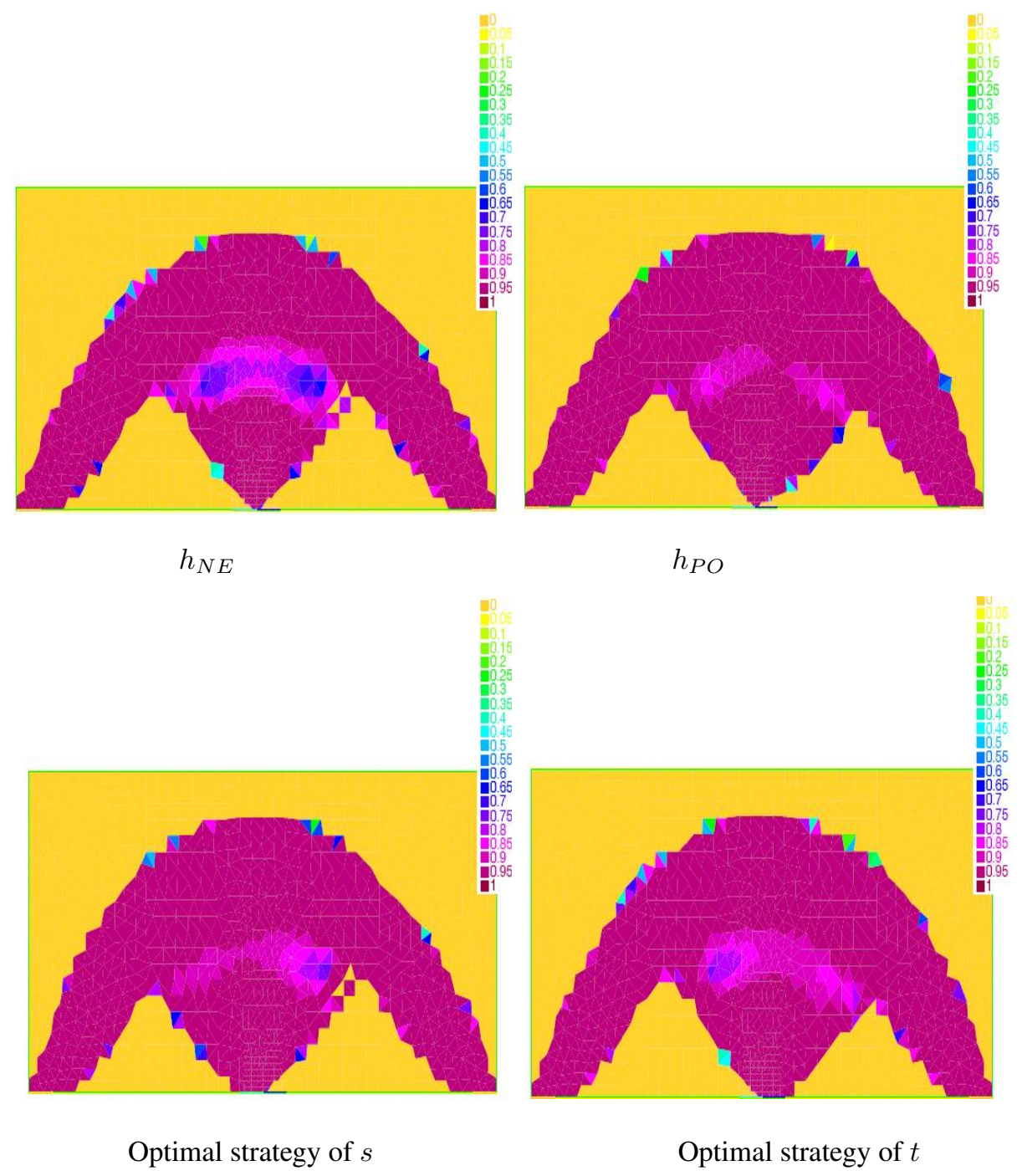

In this case, we remark that the obtained optimal shapes by Nash and Pareto are similar. 


\subsubsection{Case $2: h(s, t)=0.5 s+0.5 t$}

\begin{tabular}{|l|c|c|}
\hline & Nash Equilibrium & Pareto optimum \\
\hline$j_{1}$ & 11.454 & 11.1429 \\
$j_{2}$ & 11.4542 & 11.1436 \\
\hline
\end{tabular}

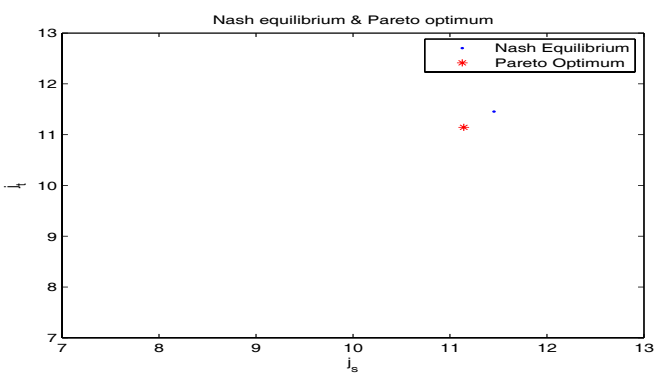

Table 2: Objective function values for Nash equilibrium and Pareto optimum $(\omega=0.5)$.

Figure 12: Pareto optimum and Nash equilibrium

Figure 13: Optimal plate. The Nash overall loop converged in 79 iterations

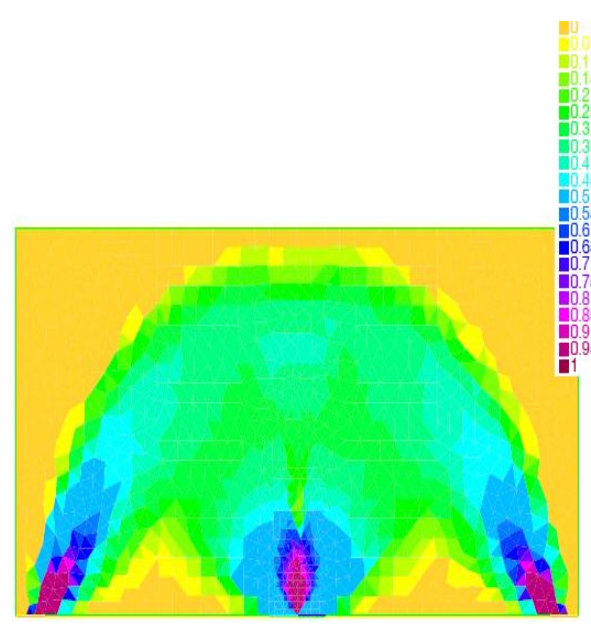

$h_{N E}$

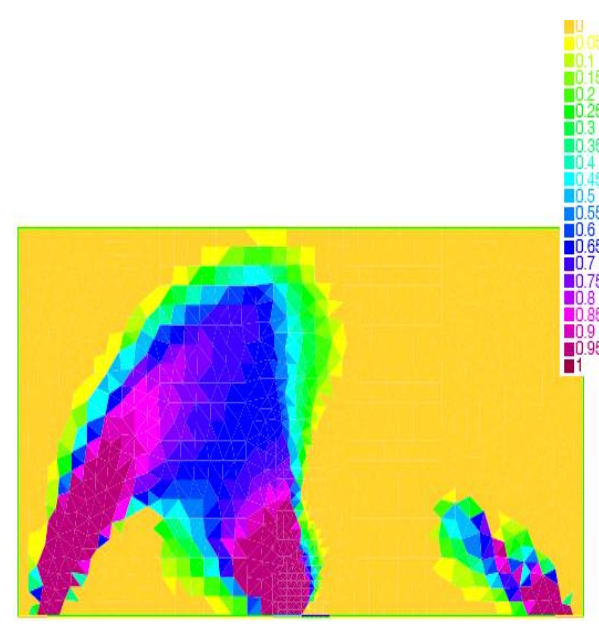

Optimal strategy of $s$

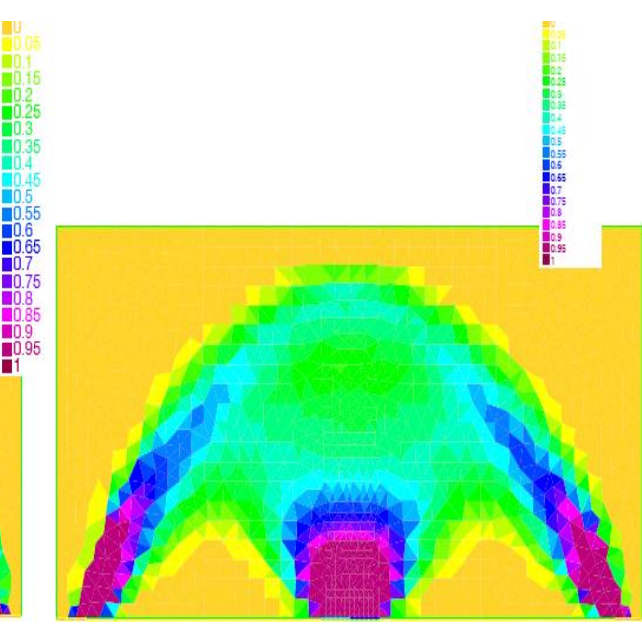

$h_{P O}$

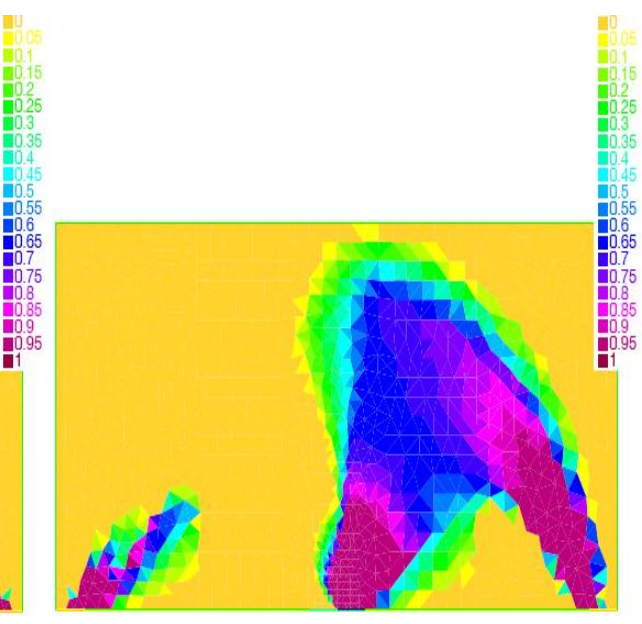

Optimal strategy of $t$ 
From the figure 13, we deduce that each player seeks to improve his criterion in his domain. The gotten shapes are symmetric.

Figure 14: Optimal plate. The Nash overall loop converged in 113 iterations
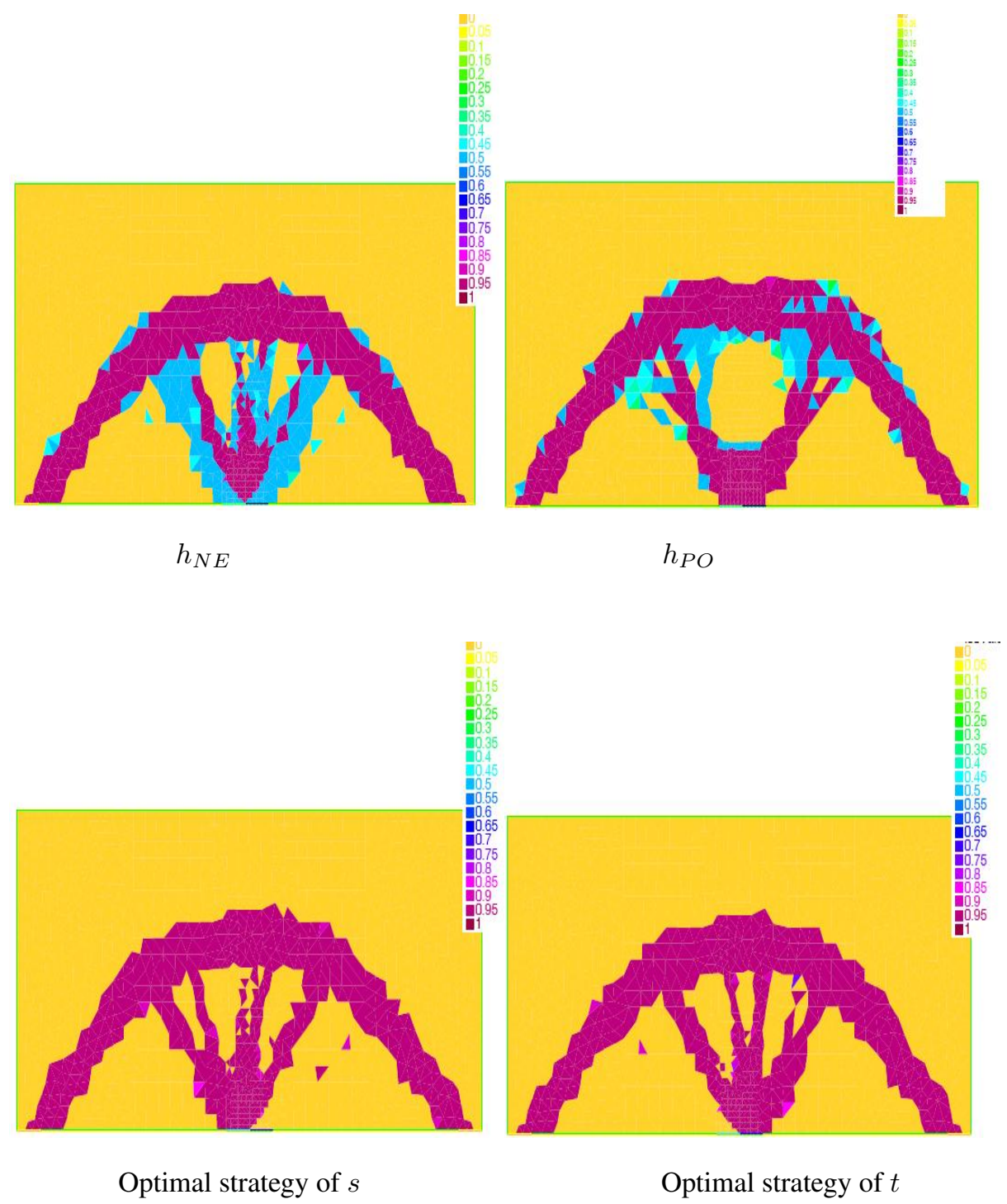

After penalization, see figure 14, the shapes associated to strategies $s$ and $t$ are generally symmetric. 


\subsubsection{Case $3: h(s, t)=s(1-t)+t(1-s)$}

\begin{tabular}{|l|c|c|}
\hline & Nash Equilibrium & Pareto optimum \\
\hline$j_{1}$ & 10.9297 & 10.7761 \\
$j_{2}$ & 10.9066 & 10.7758 \\
\hline
\end{tabular}

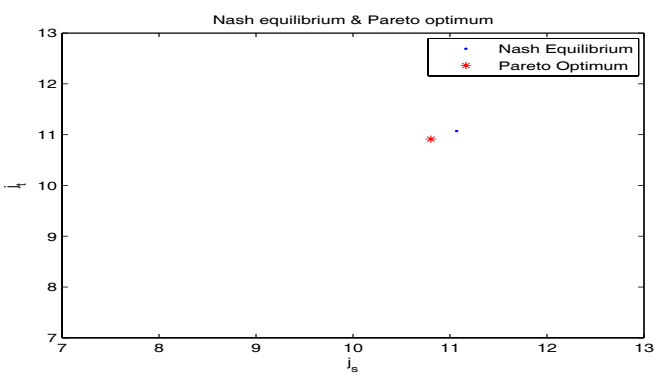

Table 3: Objective function values for Nash equilibrium and Pareto optimum $(\omega=0.5)$.

Figure 15: Pareto optimum and Nash equilibrium

Figure 16: Optimal plate. The Nash overall loop converged in 170 iterations

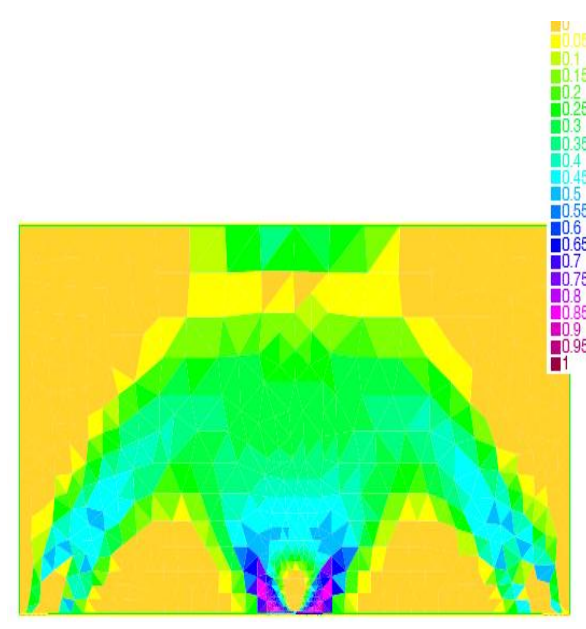

$h_{N E}$

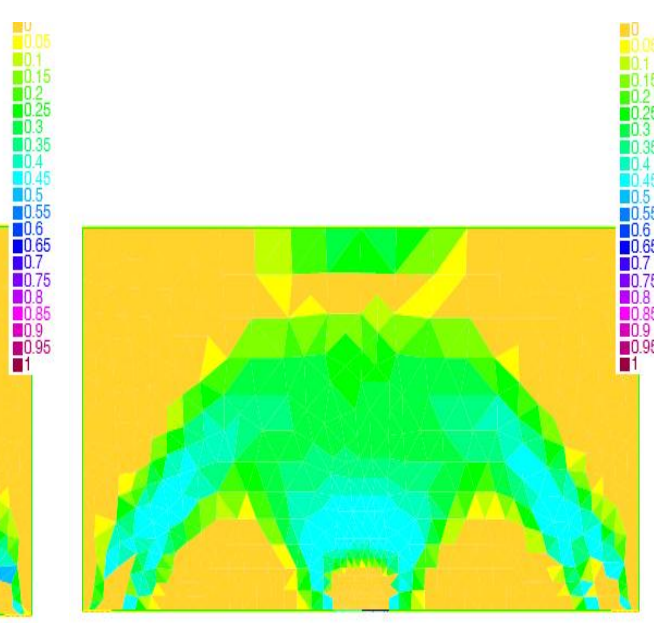

$h_{P O}$

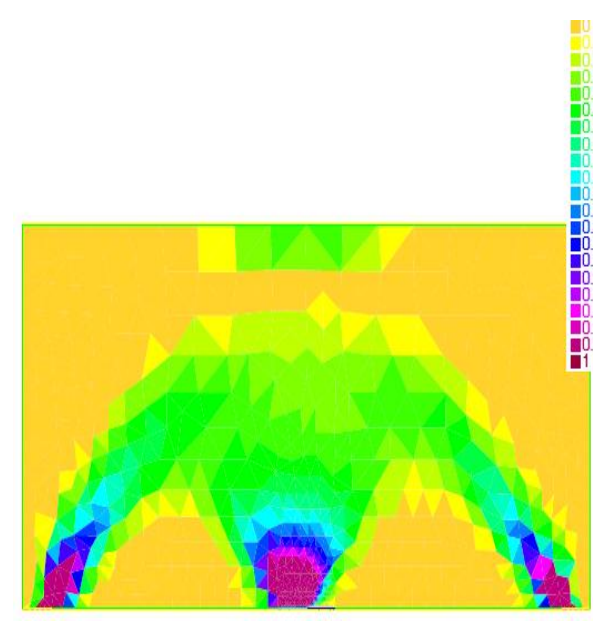

Optimal strategy of $s$

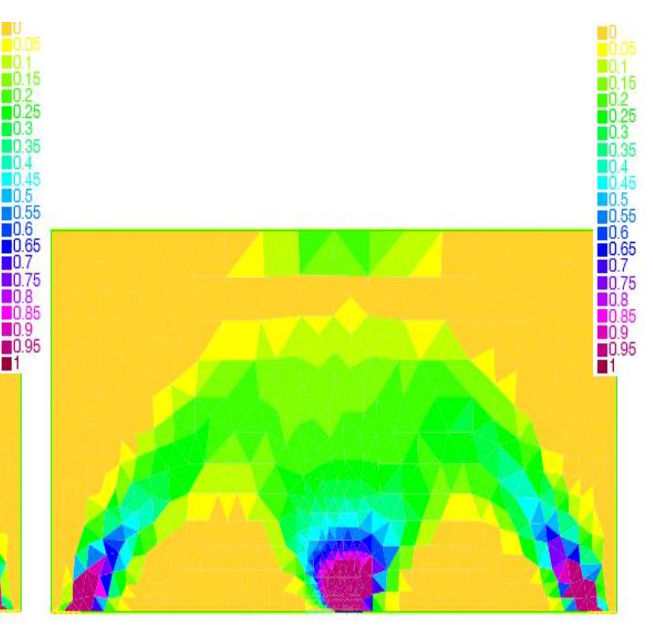

Optimal strategy of $t$ 
Figure 17: Optimal plate. The Nash overall loop converged in 191 iterations

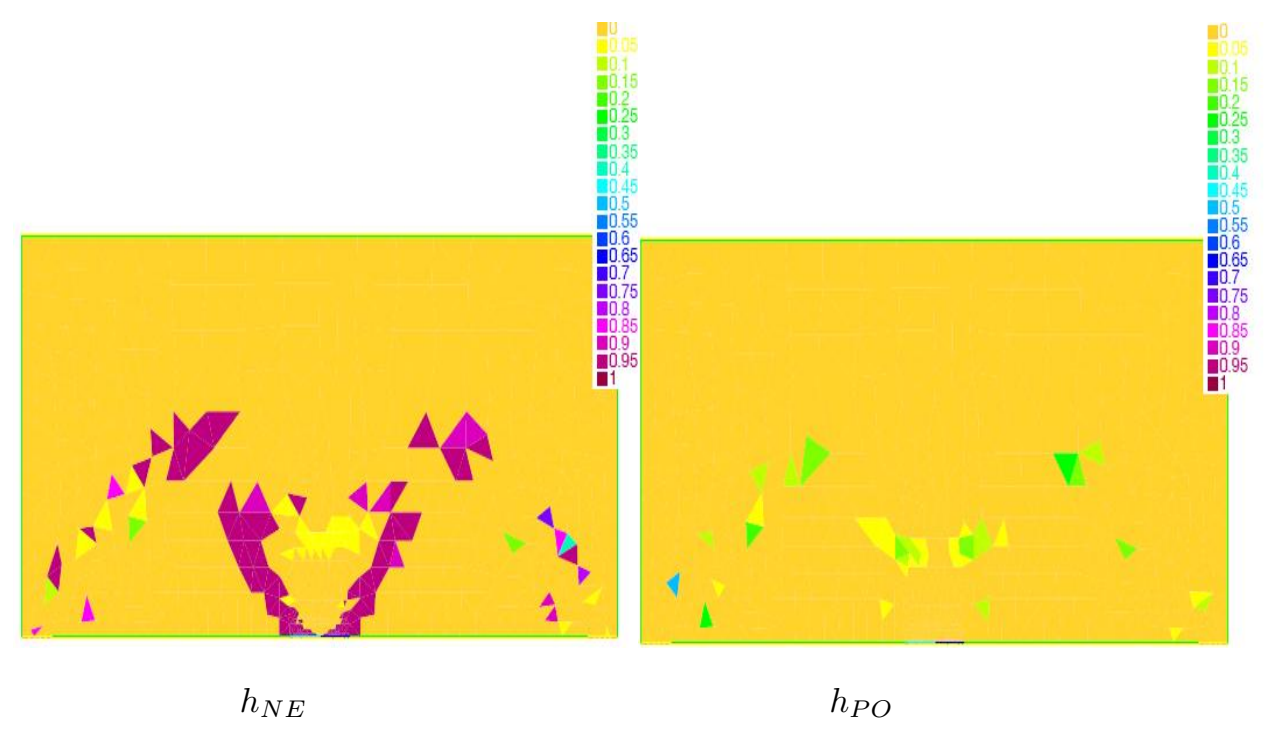

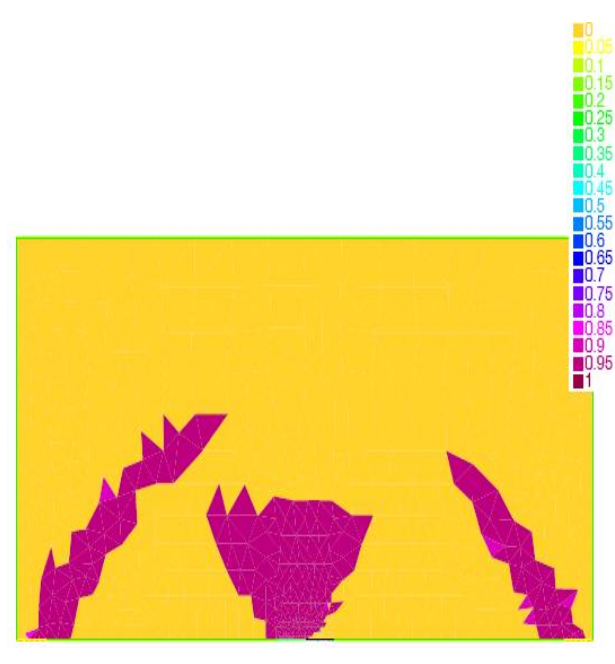

Optimal strategy of $s$

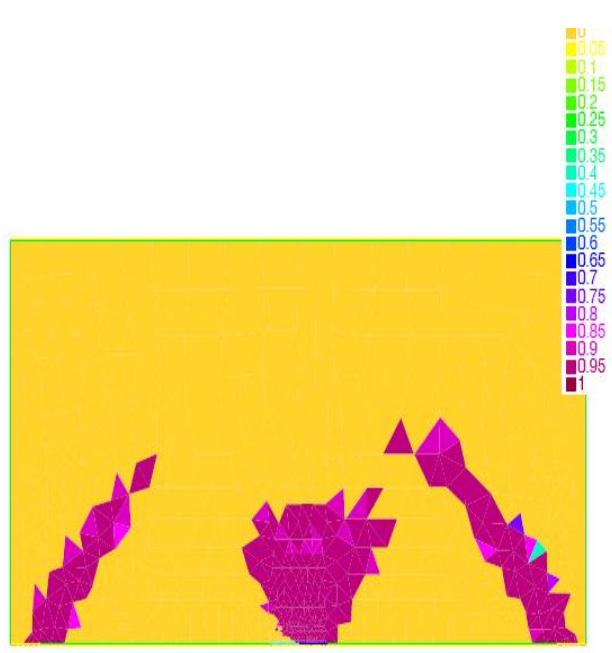

Optimal strategy of $t$

In this case, the obtained shapes are almost negligible because the penalization step of the function $h(s, t)$ is almost nil after the convergence.

\subsection{Numerical example : cantilever}

Our second example is the well-known cantilever problem which is fixed on the left wall, and supports horizontal and vertical distributed units on the middle of the right wall(see figure 18). 
Figure 18: Boundary conditions: multiple loads case

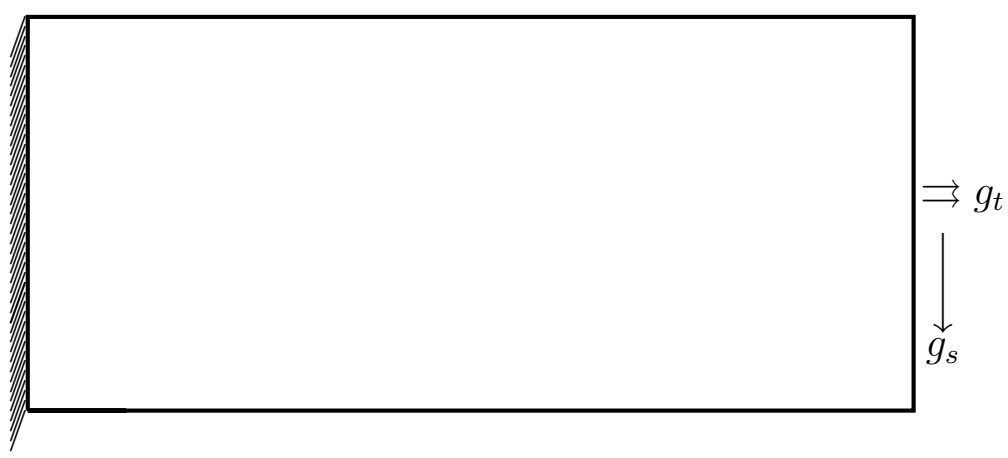

We begin with single loads, minimal compliance problems, i.e. we minimize the compliance :

$$
\min _{h} j(h)
$$

where

$$
j(h)=\int_{\Gamma_{N}} g u d s, \text { where } u \text { solves: }(2.1), \text { and } g=g_{s} \text { or } g=g_{t} .
$$

Figure 19: Optimal cantilever. single loads

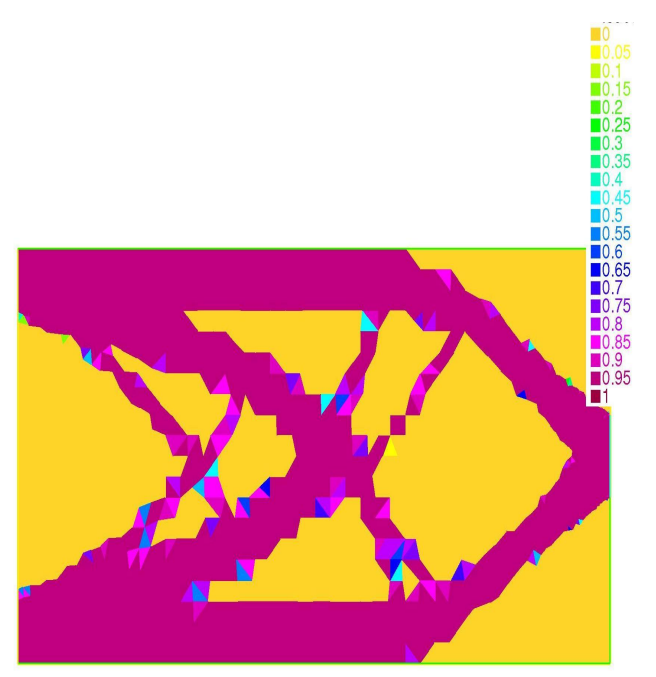

$$
g=g_{s}=(0,-1)
$$

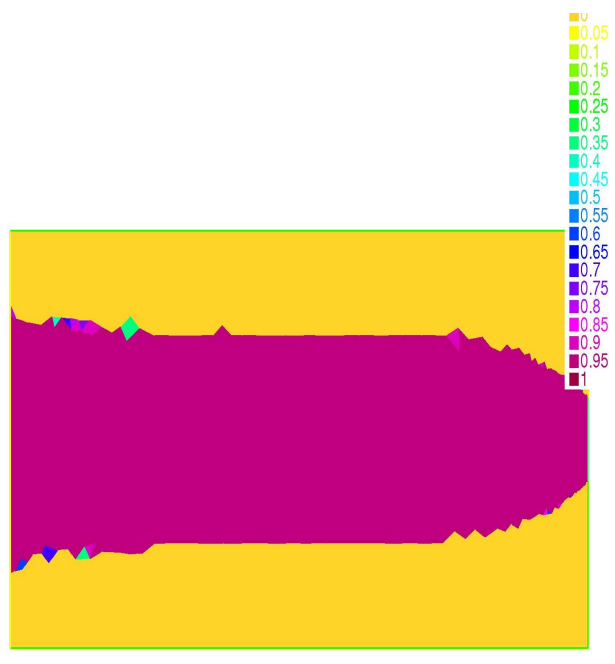

$$
g=g_{t}=(-1,0)
$$

In what follows, we apply two loads $g_{s}$ and $g_{t}$ and we present results obtained using Nash game and Pareto optimum for different strategies. 


\subsubsection{Case $1: h(s, t)=s t$}

Figure 20: Optimal cantilever. The Nash overall loop converged in 79 iterations

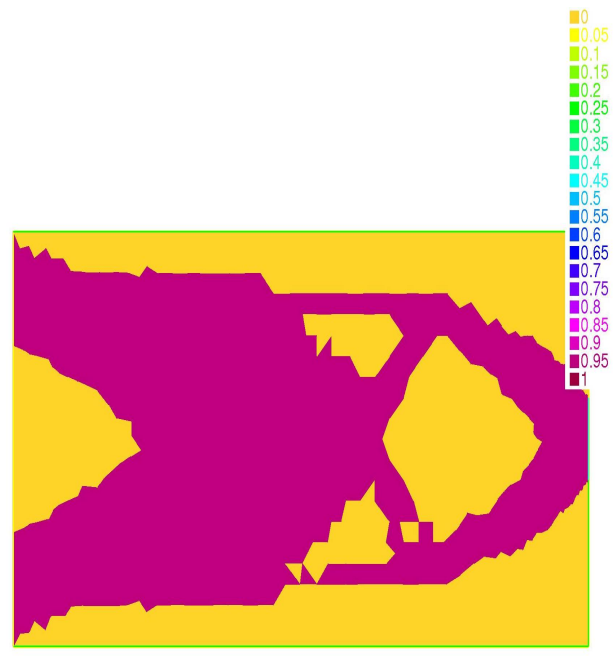

$h_{N E}$

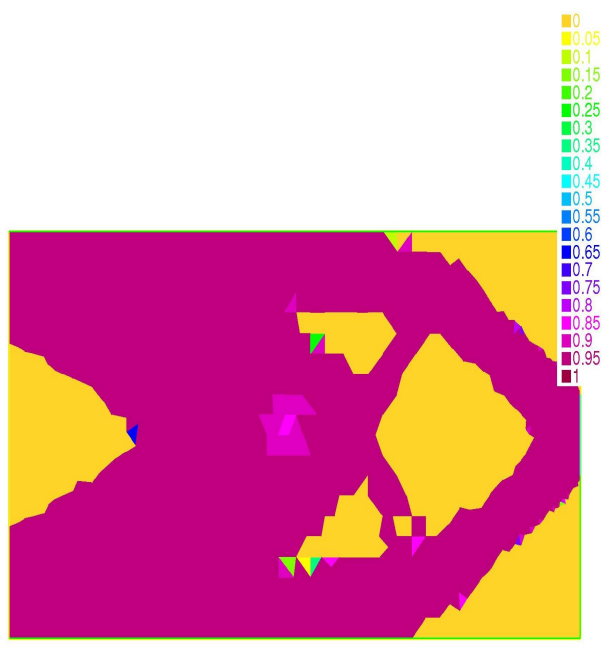

Optimal strategy of $s$

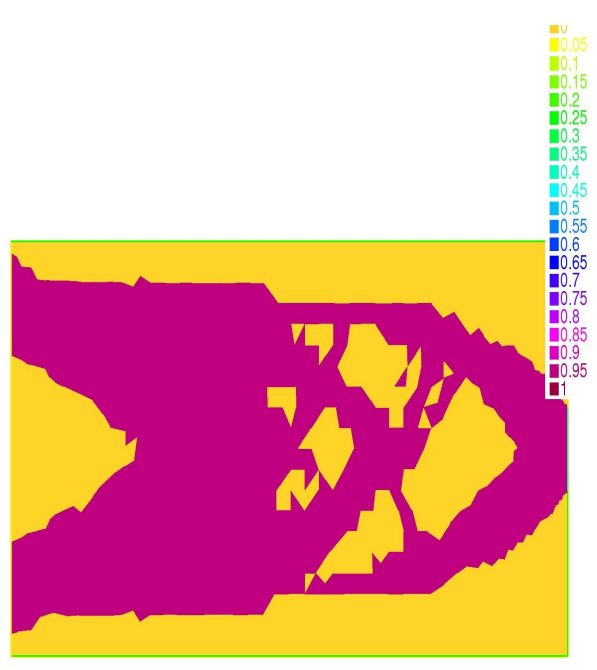

$h_{P O}$

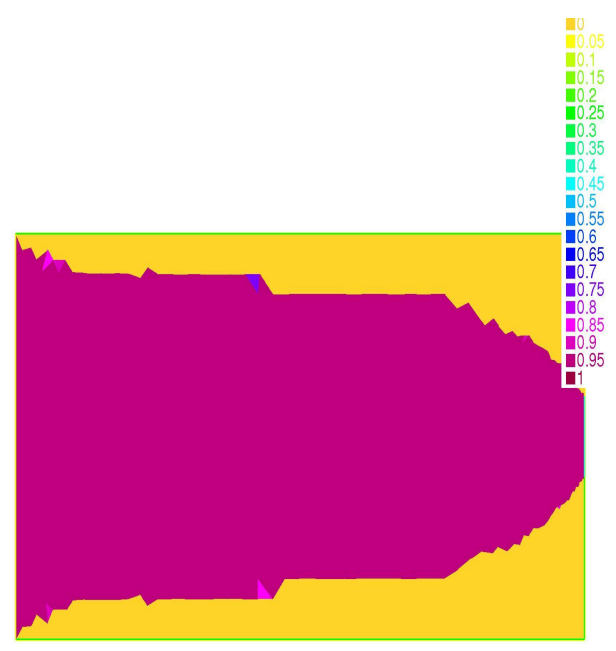

Optimal strategy of $t$

In this case, our numerical experiments show that the improvement is not sensitive since the optimal shapes are the same than those obtained by Pareto. 


\subsubsection{Case $2: h(s, t)=0.5 s+0.5 t$}

Figure 21: Optimal cantilever. The Nash overall loop converged in 91 iterations

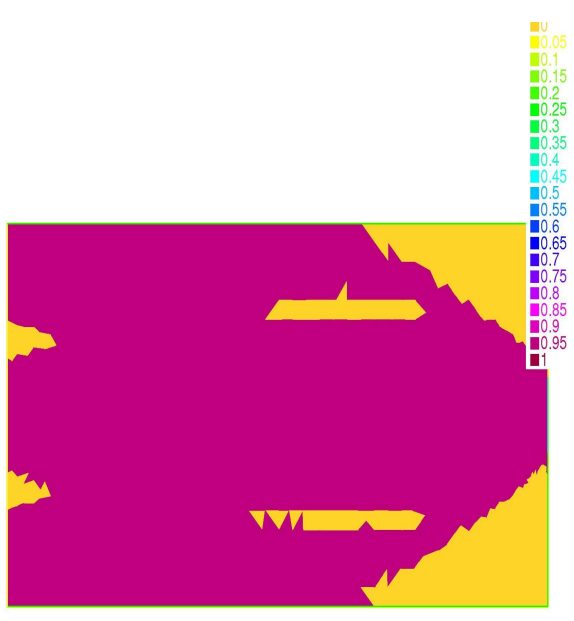

$h_{N E}$

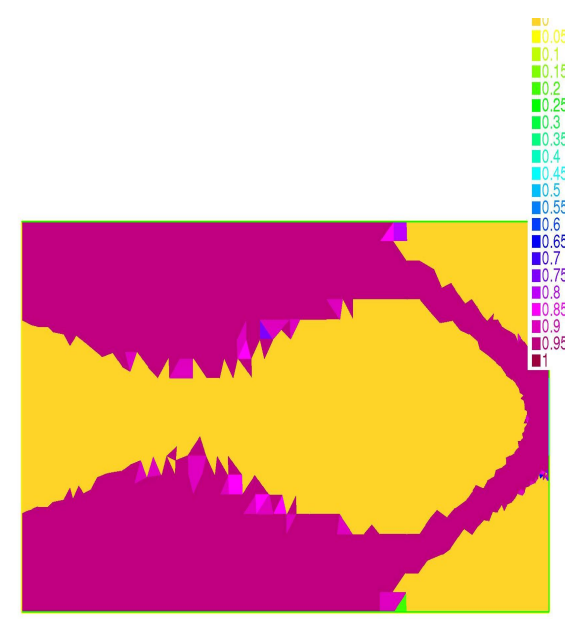

Optimal strategy of $s$

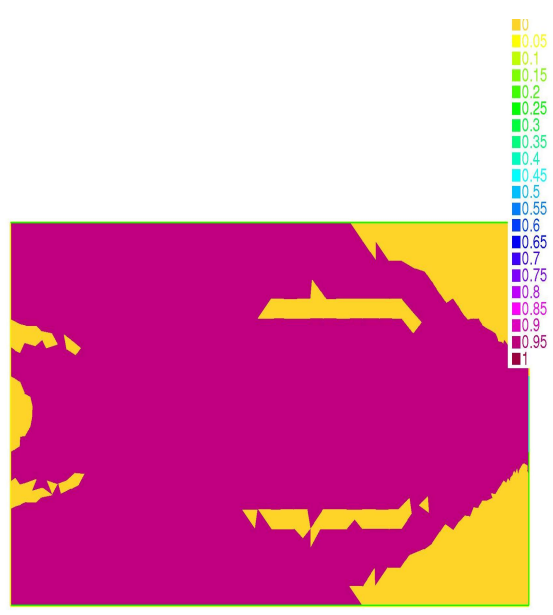

$h_{P O}$

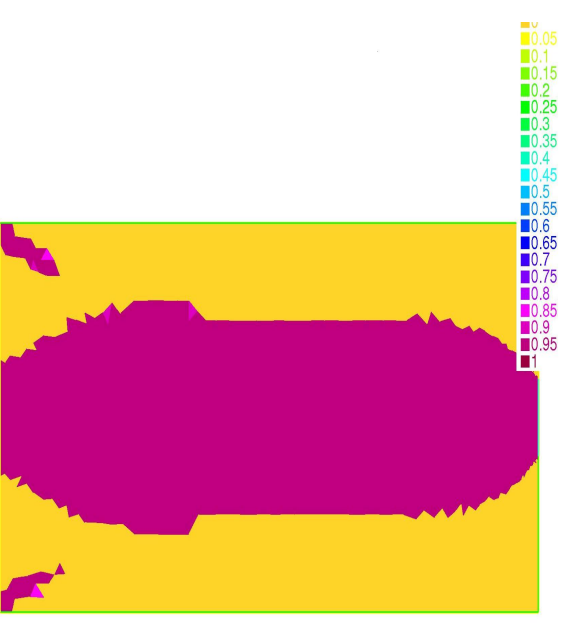

Optimal strategy of $t$

The result displayed obtained by nash is very similar to the best one in Pareto, and the objective function takes the same value. 


\subsubsection{Case $3: h(s, t)=s(1-t)+t(1-s)$}

Figure 22: Optimal cantilever. The Nash overall loop converged in 127 iterations

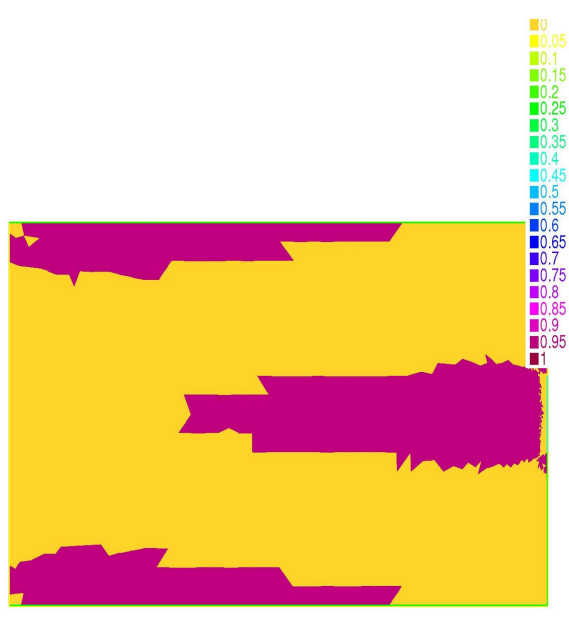

$h_{N E}$

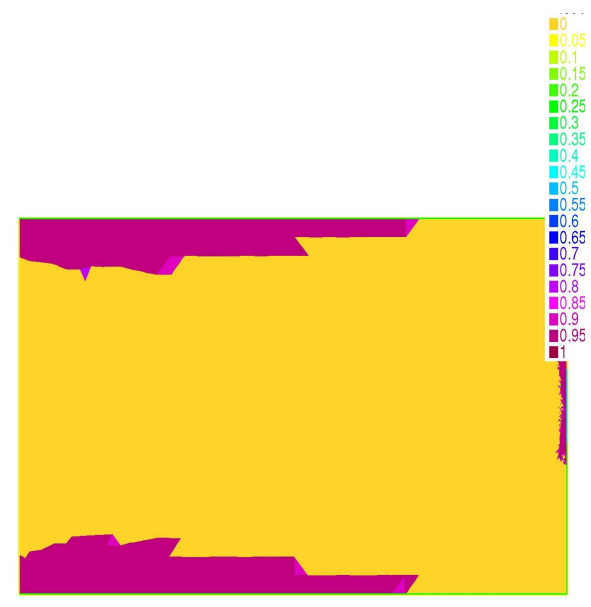

Optimal strategy of $s$

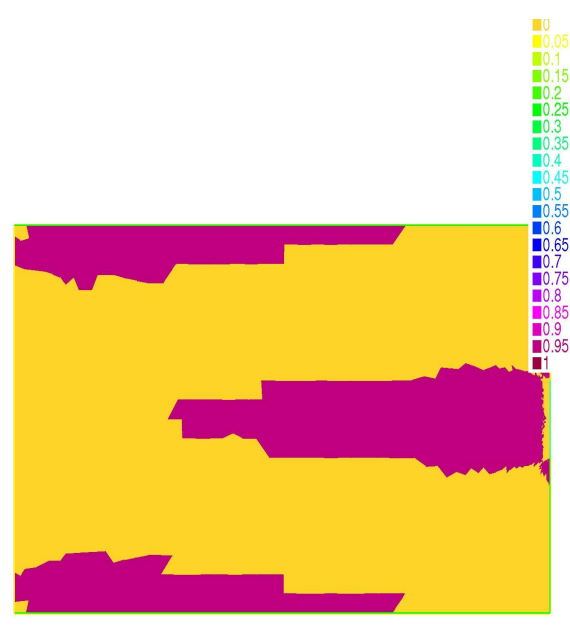

$h_{P O}$

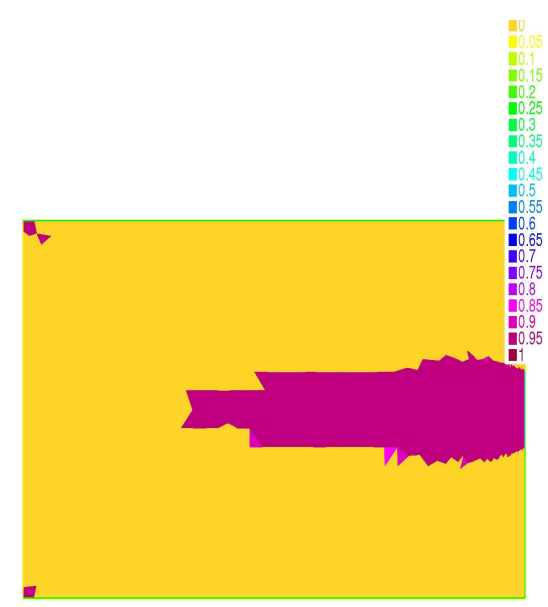

Optimal strategy of $t$

In this case, the resulting material distribution is not relevant from the mechanical point of view.

\section{Conclusion}

In this paper, we have presented and compared two approaches for solving a structural optimization problem, where we minimize the thickness of a plate subjected to two loads applied separately, on two parts of the plate. The aim of this work was the study of different strategies for splitting thickness of the plate in multidisciplinary topology optimization. We have splitted the thickness of 


\section{R. Aboulaich et al.}

material to two strategies, and we have computed the Nash equilibrium associated to this strategies, then we have compared this equilibrium with Pareto optimum. In the three cases, we observe that cases 1 and 2 give the real shapes, on the other side the case 3 give a shape which is different to the initial one. In the all three cases, for a specified value of $w$, we have obtained a Nash equilibrium close to the Pareto optimum for the choice of weights $=0.5$.

Acknowledgements/ This work was supported by Action Intégrée Franco-Morocaine MA/05/115, COADVISE/SCo $\mu$ project and the LIRIMA Laboratory.

Aboulaich, R., Habbal, A., Moussaid, N. (2010a). Split of an optimization variable in game theory, Math. Model. Nat. Phenom(MMNP), Vol. 5, No. 7, pp. 106-111.

Aboulaich, R., Habbal, A., Moussaid, N. (2010b).Optimisation multicritère : une approche par partage des variables, ARIMA, vol. 13 (2010), pp. 77-89.

Allaire, G. (2007). Conception optimale de structures, Mathématiques et Applications, vol 58.

Allaire, G. (2001). Shape optimization by the homogenization method, Springer Verlag, New York.

Allaire, G., F. d., Jouve, F., Toader, A. M. (2004). Structural optimization using topological and shape sensitivity analysis via a level-set method, Internal report no. 555, CMAP Ecole Polytechnique.

Allaire, G., Pantz, O. (2005). Structural Optimization whith Freefem++, R.I 586.

Aubin, J.P. (1979). Mathematical methods of game and economic theory. North-Holland Publishing Co. Amsterdam, New York.

Bendsøe, M.P., Kikuchi, N. (1988). Generating optimal topologies in structural design using a homogenization method, Comput. Methods Appl. Mech. Eng. 71, 197224.

Duysinx, P., Bendsøe, M.P.(1988). Topology optimization of continuum structures with local stress constraints, Int. J. Numer. Methods Eng. 43, 14531478.

Habbal, A. (2005). A topology Nash game for tumoral antangiogenesis. Struct Multidisc Optim 30:404-412.

Habbal, A. (2004). Finite Element Convergence of optima, report.

Habbal, A., Petersson, J., Thellner, M. (2004). Multidisciplinary topology optimization solved as a Nash game. Int, J. Numer. Meth. 61:949-963.

Nash, J.F. (1951). Non-cooperative Games, Annals of Mathematics.

Amstutz, S., Andrä, H. (2002). A new algorithm for topology optimization using a level-set method. Reserach report number 4543, INRIA. 